\title{
Austrian Vegetation Database
}

\author{
Wolfgang Willner, Christian Berg \& Paul Heiselmayer
}

\begin{abstract}
The aim of the Austrian Vegetation Database (GIVD ID EU-AT-001) is to gather computerised phytosociological information (vegetation relevés) from the territory of Austria. The data are stored in TURBOVEG format. Large parts of the database have been gathered between 1997 and 1999 and between 2001 and 2003 (partly funded by the Austrian Academy of Science). However, the database is still far from being complete. Especially, ruderal and weed communities and (semi-)aquatic vegetation is only insufficiently covered yet. Due to the absence of funding, progress is rather slow at the moment.
\end{abstract}

Keywords: Austria; phytosociology; TURBOVEG.

\section{Austrian Vegetation Database}

Scope: The aim of the Austrian Vegetation Database is to gather computerized phytosociological information (vegetation relevés) from the territory of Austria.

Status: ongoing capture

Period: 1926-2011

Database manager(s): Wolfgang Willner (wolfgang.willner@vinca.at)

Owner: (private)

Web address: http://vegedat.vinca.at/

Availability: free upon request

Online upload: no

Online search: no

Database format(s): TURBOVEG

Export format(s): TURBOVEG, MS Access, Excel, plain text file, Cornell

Publication: [NA]

Plot type(s): normal plots

Plot-size range: $0.1-4000 \mathrm{~m}^{2}$

Non-overlapping plots: 42,000

Total plot observations: 42,000

Estimate of existing plots: 100,000

Number of sources: 550

Completeness: $42 \%$

Valid taxa: 4,151

Countries: AT: $100.0 \%$

Forest: [NA] — Non-forest: [NA]

Guilds: all vascular plants: $100 \%$; bryophytes (terricolous or aquatic): $20 \%$; lichens (terricolous or aquatic): $5 \%$

Environmental data: altitude: $83 \%$; slope aspect: $63 \%$; slope inclination: $65 \%$; microrelief: $5 \%$; soil depth: $0 \%$; surface cover other than plants (open soil, litter, bare rock etc.): $7 \%$

Performance measure(s): cover: $100 \%$

Geographic localisation: GPS coordinates (precision $25 \mathrm{~m}$ or less): $3 \%$; point coordinates less precise than GPS, up to $1 \mathrm{~km}$ : $18 \%$; small grid (not coarser than $10 \mathrm{~km}$ ): $58 \%$; political units or only on a coarser scale $(>10 \mathrm{~km}): 124 \%$

Sampling periods: 1920-1929: 0.1\%; 1930-1939: 1.4\%; 1940-1949: 1.3\%; 1950-1959: 4.2\%; 1960-1969: 7.0\%; 1970-1979: 18.0\%; 1980-1989: 34.0\%; 1990-1999: 25.0\%; 2000-2009: 8.0\%; 2010-2019: $1.0 \%$

Information as of 2012-07-12; further details and future updates available from http://www.givd.info/ID/EU-AT-001

Wolfgang Willner* (wolfgang.willner@vinca.at)

VINCA, Giessergasse 6/7, 1090 Wien, AUSTRIA

Christian Berg (christian.berg@uni-graz.at)

Institut für Pflanzenwissenschaften, Karl-Franzens-Universität Graz, Holteigasse 6, 8010 Graz, AUSTRIA

Paul Heiselmayer (paul.heiselmayer@sbg.ac.at)

Fachbereich Organismische Biologie, Universität Salzburg, Hellbrunnerstr. 34, 5020 Salzburg, AUSTRIA

*Corresponding author 